\title{
Diseño preliminar de la configuración de cabina para la aeronave de entrenamiento primario Phaeton PT-01
}

\author{
Juan Sebastián Gaviria García \\ Ingeniero Aeronáutico, Universidad Pontificia Bolivariana. \\ Medellín, Colombia \\ juanbatian@gmail.com
}

\author{
Elías E. Meriño Díaz \\ Ingeniero Aeronáutico, Universidad Pontificia Bolivariana. \\ Medellín, Colombia \\ eliase@themerinos.com
}

\begin{abstract}
Resumen- El espacio en cabina de una aeronave influye directamente en las capacidades de la tripulación a la hora de realizar su trabajo. La comodidad, el ambiente, la iluminación, la forma en que se presenta toda la información de la aeronave en los instrumentos son algunos de los factores que son considerados en toda situación de vuelo. La metodología de diseño preliminar de este espacio de cabina y su interacción con la tripulación basada en procesos de diseño centrados en el ser humano, será el principal tópico de este artículo.
\end{abstract}

Palabras clave - Ergonomía, Antropometría, Visibilidad, Controles de Mando, Instrumentos de Vuelo, Seguridad, Diseño Preliminar.

\begin{abstract}
The cockpit in an aircraft directly affects the capabilities of the crew to do their jobs. Comfort, atmosphere, lighting condition, the way that all the information from the aircraft is shown in the instruments are some of the factors that are considered in every flight situation. The methodology for the preliminary design of the cockpit and its interaction with the crew based on a design process centered on human beings will be the main topic of this article.
\end{abstract}

Keywords- Ergonomics, Anthropometrics, Visibility, Flight Controls, Flight Instruments, Safety, Preliminary Design.

\section{INTRODUCCIÓN}

Actualmente, Colombia tiene un elevado potencial en diseño de aeronaves que se refleja por el gran posicionamiento que tienen los ultralivianos colombianos en el mercado. A pesar de esto, el diseño de las cabinas de los mismos es bastante sencillo, y debido a que no se encuentran documentos elaborados en el país que hagan referencia a un proceso de diseño de cabinas para estas aeronaves, puede pensarse que dicho diseño está basado en información empírica de los diseños elaborados por los fabricantes extranjeros, sin llegar a una metodología de diseño adecuada para satisfacer los parámetros de confort y seguridad para los tripulantes. Por esta razón, se pretende en este artículo desarrollar dicha metodología y aplicar un nuevo proceso de diseño llamado Proceso de Diseño Centrado en el Ser Humano, el cual permite implementar las últimas tecnologías en términos de análisis ergonómico y aviónica digital para su uso en aeronaves que requieren certificación para su operación, como es el caso del Phaeton PT-01.

Este proceso inicia con el estudio antropométrico de la población colombiana que usará la aeronave. Con esto se realiza el análisis ergonómico, el cual incluye la interacción del tripulante con sus instrumentos y controles en su posición de vuelo, junto con los factores de seguridad que involucra este análisis en caso de accidente. La metodología de diseño finaliza con la ubicación y distribución de instrumentos junto con el dimensionamiento del panel y del espacio interior de cabina en la aeronave. Adicionalmente se seleccionan asientos, instrumentos, controles de mando y otros subsistemas como la seguridad y el acondicionamiento de aire.

\section{PROCESO DE DIMENSIONAMIENTO DE CABINA DE VUELO}

El diseño de una aeronave consta de tres etapas básicas: diseño conceptual (donde se diseñan y escogen las características básicas del aparato), diseño preliminar (que presenta mayor grado de exactitud en el diseño y en el que se congelan y determinan muchas de las características diseñadas, después de hacer cambios y mejoras sobre el diseño conceptual) y diseño detallado (en el cual, como su nombre lo indica, se especifican los detalles de todo el diseño, cuáles y como serán todos los componentes de la aeronave y los procesos de manufactura que se usarán).

Este artículo trata del diseño preliminar de la cabina de vuelo para una aeronave específica: el 
Phaeton PT-01, aeronave de ala fija para el entrenamiento primario, de la cual se hizo previamente el diseño conceptual (elaborado por los autores de este artículo junto con el ingeniero aeronáutico Sergio A. Arias).

A partir de los resultados del diseño conceptual de la cabina para el Phaeton se hace el diseño preliminar de dicha cabina, se comienza con el dimensionamiento del espacio interior.

En la etapa conceptual del diseño se definió el ancho máximo de la cabina del avión con una dimensión de $1.32 \mathrm{~m}$. El ancho de cabina es muy importante en este avión puesto que se diseña con una configuración de distribución de asientos lado-alado, por lo que se requiere de suficiente espacio para garantizar comodidad a la tripulación. Esta dimensión se comparó con la existente en aviones del mismo tipo ya existentes, con el fin de decidir si el ancho de cabina podía congelarse o debía modificarse. La comparación se hizo con los datos tomados en aeronaves Cessna y Piper. Al notarse que el ancho de cabina del Phaeton es mayor que el de las aeronaves en operación se decidió fijar esta dimensión en el diseño.

\section{A. Análisis ergonómico}

Para el dimensionamiento se hace necesario elaborar un dummy con todas las dimensiones antropométricas correspondientes a la tripulación, lo que implica la selección previa de la población para la cual se diseña el avión.

Al Buscar que el producto pueda ser usado por un espectro muy amplio de la población se usan en los procesos de diseño rangos de dimensiones antropométricas para establecer los valores mínimo y máximo de ajuste que garantizan cómodo acceso a la máquina. Los valores antropométricos suelen presentarse organizados en percentiles.

El dummy se dimensionó de acuerdo al estudio antropométrico realizado para Colombia en 1995 por parte de la Facultad Nacional de Salud Pública de la Universidad de Antioquia y el Instituto de Seguros Sociales[1]. Los resultados del estudio corresponden a una muestra de 2100 personas laboralmente activas en Colombia y cuyas edades están entre los 20 y 59 años de edad, 1315 de sexo masculino y 785 de sexo femenino. Este estudio muestra que la población de mayor estatura corresponde a las personas entre los 20 y 29 años de edad, definiéndose ésta como población de diseño.
Se parte de los requerimientos antropométricos para la tripulación (1.65-1.95m de estatura y 0.85$1.02 \mathrm{~m}$ de altura de tronco), se selecciona el rango de estaturas comprendido entre el percentil 5 que corresponde a una estatura de $1.59 \mathrm{~m}$ y el percentil 95 que se refiere a la estatura de $1.81 \mathrm{~m}$. El percentil 95 se usa para la elaboración del dummy que servirá como piloto de tamaño intermedio y que definirá las posiciones neutras de sillas, pedales y demás controles en la cabina así como otras dimensiones importantes. Para determinar las posiciones de ajuste, mínima y máxima, de las sillas y mandos de control se utilizan respectivamente el percentil 5 y la estatura de $1.95 \mathrm{~m}$ determinado por los requerimientos y que escapa a los percentiles antropométricos.

Los tres dummies se modelaron en AutoCAD ${ }^{\circledR}$ de forma tal que se permite la variación de su posición mediante articulaciones y facilite el dimensionamiento y análisis de alcance de los controles y confort para las diferentes posturas.

Una vez elaborado cada dummy, el paso siguiente es ubicar el tripulante sentado donde su punto de referencia es el ojo, para no restringir su visibilidad hacia afuera. El Dr. Jan Roskam [2] recomienda unas dimensiones geométricas para la configuración de cabina y ubicación de asientos, que establecen espacios adicionales para permitir que las variaciones del cuerpo humano de persona a persona no afecten la configuración de cabina. Las recomendaciones geométricas limitan las variaciones corporales a $+/-15 \mathrm{~cm}$ en longitud de brazo, +/- $20 \mathrm{~cm}$ en longitud de piernas y +/- 12 $\mathrm{cm}$ en distancia del asiento al ojo.

FIG. 1. UBICACIÓN DEL DUMMY SEGÚN ERGONOMÍA

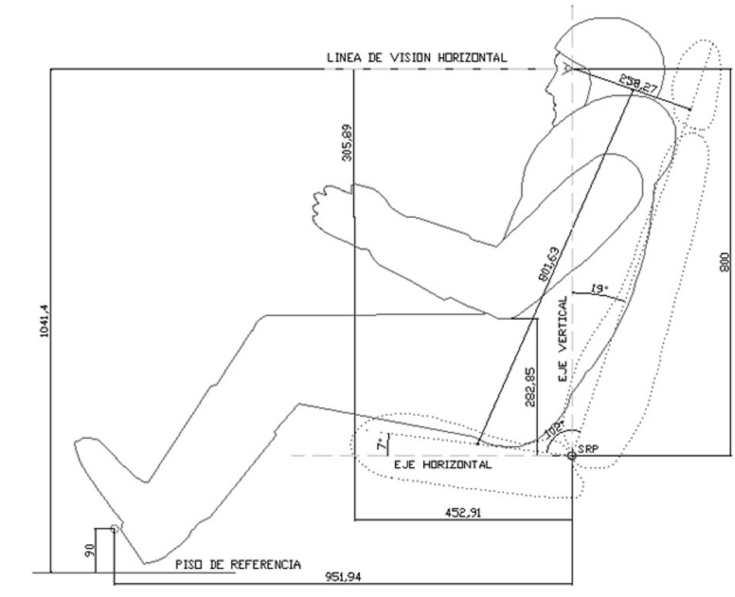

Fuente: Los autores 
Para determinar la posición neutra de la silla, es decir, aquella posición en la cual la silla no ha sido ajustada en altura hacia arriba o abajo ni en distancia horizontal que la separa del panel de instrumentos, se ubica el dummy percentil 95 en la silla teniendo como referencia la posición vertical y horizontal del ojo respecto al piso de cabina y a los pedales respectivamente. Una vez ubicado así, queda determinado el Punto de Referencia de la Silla o Seat Reference Point (SRP) que es el vértice de unión entre el asiento y el espaldar, en sus líneas medias. Ahora, los ajustes de las sillas para los diferentes tamaños de tripulación están dados por las variaciones en altura y distancia horizontal que presente el SRP. La Fig. 1 muestra al dummy de percentil 95 ubicado como se mencionó.

Las limitaciones de movimiento y alcance que tiene el cuerpo humano son de gran importancia en el dimensionamiento preliminar de cabina ya que instrumentos y controles de vuelo no pueden ubicarse de manera que se superen estas restricciones corporales. Por eso, para este análisis, se tienen en cuenta las limitaciones de flexión dorsal del tobillo para los pedales; las limitaciones del campo visual, lo cual se explica en la sección $B$; y las limitaciones en el movimiento de los brazos para el alcance de los controles y el panel.

Para el caso del tobillo, los ángulos límites son de $20^{\circ}$ de inclinación del pie hacia arriba y $40^{\circ}$ hacia abajo, lo que limita el dimensionamiento de los pedales en su ángulo de inclinación normal y ángulo de inclinación con el freno aplicado. Estos valores varían un poco según la población y el percentil. Para el Phaeton se escoge una posición de $26^{\circ}$ para el pedal sin aplicación de freno y $40^{\circ} \mathrm{con}$ aplicación de freno.

Se modelan también los diagramas de alcance que consisten en los contornos horizontales de movimiento de los brazos en posición sentada con el arnés de seguridad amarrado. Esto permite verificar el punto máximo que la tripulación pueda alcanzar con un solo movimiento del brazo sin realizar un esfuerzo consciente. La Fig. 2 ilustra estos diagramas de alcance para estaturas de $1.60 \mathrm{~m}, 1.81 \mathrm{~m}$ y $1.95 \mathrm{~m}$. Para la población incluida en el percentil 5, el alcance se ve reducido en un $13 \%$ y para una población de $1.95 \mathrm{~m}$, el mismo se ve aumentado en un 6\%. Estos porcentajes se toman respecto a la población del percentil 95, la cual constituye la población de diseño. Cabe re- saltar que el diagrama de alcance se modela para cada mano: izquierda y derecha, que son simétricas entre sí.

FIG. 2. DIAGRAMAS DE ALCANCE PARA LA TRIPULACIÓN

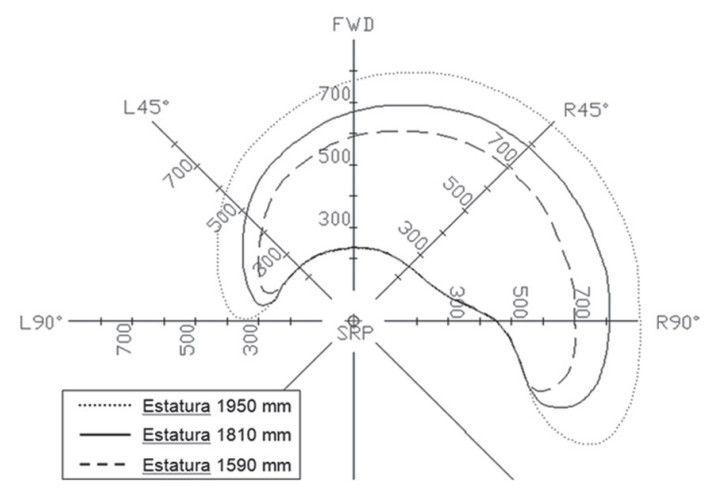

Fuente: Los autores

Una vez definido el SRP y elaborados los diagramas correspondientes de alcance, se hicieron coincidir el SRP actual con el que se definió en la etapa conceptual de diseño, y a partir de ello se ubican los pedales y el panel de instrumentos de manera tal que el dummy tenga cómodo alcance de ellos. Seguidamente se realizaron las pequeñas variaciones necesarias en las posiciones de SRP, pedales y panel. La distancia a la cual se ubica el panel y los ajustes posibles de los pedales son probados con los diagramas de alcance de cada uno de los dummies ubicados en su respectivo SRP. Para terminar de definir la posición de cada piloto en la cabina hace falta hacer el análisis de visibilidad.

\section{B. Análisis de visibilidad}

El análisis de visibilidad se divide en dos: visibilidad interna y visibilidad externa. La visibilidad interna de la cabina implica poder visualizar completa y cómodamente el panel de instrumentos y los controles de mando, mientras que la visibilidad al exterior hace referencia a la posibilidad de visualizar todo aquello que está por fuera de la aeronave tanto en vuelo como en tierra.

Para la visibilidad externa, el análisis se hizo de acuerdo con las recomendaciones de diseño de Roskam[2] y la FAA[3], los cuales definen los ángulos mínimos de visibilidad que debe poseer el piloto tanto en sentido horizontal como vertical. 
El campo de visibilidad es medido en dos planos perpendiculares: un plano horizontal a la altura de los ojos del piloto en el cual se miden los ángulos de visión azimutal; y un plano vertical que pasa por el punto de diseño del ojo (punto ubicado en el medio de los dos ojos del piloto) y el eje de rotación de la cabeza. Sobre este eje, ubicado justo detrás del punto de diseño del ojo (O) se ubica otro punto llamado C. Los ángulos de visión se miden entre vectores que surgen desde el punto $C$ para la medición horizontal y entre vectores sobre el plano vertical que surgen desde el punto 0 para la visibilidad vertical. Ambas mediciones angulares (vertical y horizontal) se analizan para el diseño a partir de curvas que presentan los rangos de visibilidad vertical que ha de existir para determinado ángulo de visibilidad horizontal, como lo muestra la Fig. 3. Estas curvas utilizadas para el diseño de cabina del Phaeton corresponden a requerimientos de visibilidad para aeronaves de categoría FAR 25. Sin embargo se utilizaron debido a que para la categoría de FAR 23 no se encontraron requerimientos de visibilidad.

FIG. 3. CURVA ENVOLVENTE DE VISIBILIDAD

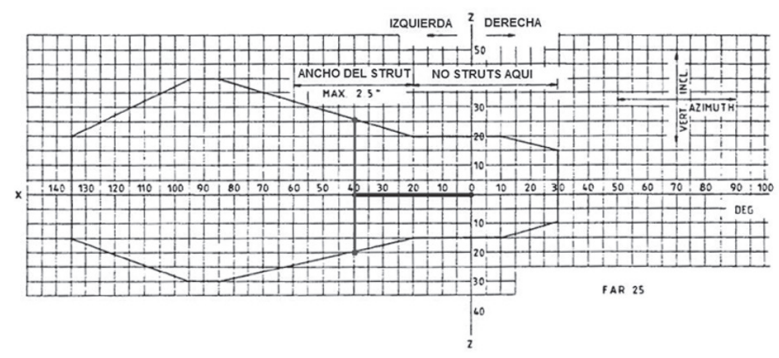

Fuente: Los autores

De acuerdo con estos perfiles de visibilidad se trazaron, mediante el AutoCAD ${ }^{\circledR}$, los rangos angulares de visibilidad horizontal y vertical desde la respectiva ubicación del ojo y del centro de rotación de cabeza para cada uno de los tres pilotos (dummies).

TABLA I

Rangos angulares definidos para cada uno de los dummies en el Phaeton

\begin{tabular}{|c|c|c|c|c|c|c|c|}
\hline $\begin{array}{c}\text { Estatura } \\
\text { Piloto }\end{array}$ & $\begin{array}{c}\text { Ángulo } \\
\text { horizontal } \\
\text { lado interior }\end{array}$ & $\begin{array}{c}\text { Ángulo } \\
\text { horizontal } \\
\text { lado } \\
\text { exterior }\end{array}$ & $\begin{array}{l}\text { Ángulo vertical } \\
\text { hacia arriba } \\
\text { (Mínimo } \\
\text { recomendado) }\end{array}$ & $\begin{array}{c}\text { Ángulo } \\
\text { vertical } \\
\text { hacia } \\
\text { abajo } \\
\text { (Mínimo } \\
\text { requerido) }\end{array}$ & $\begin{array}{c}\text { Ángulo } \\
\text { horizontal } \\
\text { de } \\
\text { obstáculo }\end{array}$ & $\begin{array}{c}\text { Ángulo } \\
\text { vertical } \\
\text { de } \\
\text { obstáculo }\end{array}$ & $\begin{array}{c}\text { Ángulo } \\
\text { sobre nariz } \\
\text { (Vertical } \\
\text { hacia } \\
\text { abajo, real) }\end{array}$ \\
\hline 1.59 m & $30^{\circ}$ & $135^{\circ}$ & $20^{\circ}$ & $15^{\circ}$ & $40^{\circ}$ & $58^{\circ}$ & $12^{\circ}$ \\
\hline $1.81 \mathrm{~m}$ & $30^{\circ}$ & $135^{\circ}$ & $20^{\circ}$ & $15^{\circ}$ & $35^{\circ}$ & $45^{\circ}$ & $12^{\circ}$ \\
\hline $1.95 \mathrm{~m}$ & $30^{\circ}$ & $135^{\circ}$ & $20^{\circ}$ & $15^{\circ}$ & $33^{\circ}$ & $40^{\circ}$ & $12^{\circ}$ \\
\hline
\end{tabular}

Fuente: Los autores

Las cuatro primeras columnas de la Tabla I tienen los mismos valores en tanto que estos son valores sugeridos. Las columnas de ángulos de obstáculo sí difieren en los valores puesto que se refieren a los ángulos en los cuales cada dummy desde su posición encuentra un obstáculo visual, que son los struts del canopy. Estos ángulos se encuentran dentro de los rangos permitidos como se muestra en la Fig. 4. El ángulo sobre nariz es el máximo ángulo real de visibilidad que tiene el piloto hacia abajo por encima de la nariz del avión, presenta una diferencia de $3^{\circ}$ respecto al mínimo recomendado.

Los rangos de visibilidad de $165^{\circ}$ horizontal y $32^{\circ}$ vertical son los rangos mínimos con que cum- ple Phaeton. Sin embargo, los pilotos de este avión contarán con rangos mucho más amplios puesto que pueden mirar más allá hacia ambos lados y hacia arriba debido al tipo de parabrisas que se implementará, el cual es una cubierta transparente tipo canopy.

El análisis que se hace para la visibilidad interna de la tripulación no se basa en diagramas de visibilidad que implican movimiento conjunto de ojos y cabeza si es requerido, sino que se define con los campos de visión primario y secundario de cada piloto, desde su posición y según la importancia de los instrumentos y controles que deba ubicar con su mirada.

El campo de visión primario (PFV por sus siglas en inglés) se refiere a los rangos de visión que alcan- 
za cada piloto tanto en sentido vertical como horizontal únicamente con el movimiento de sus ojos. Dentro del PFV han de estar ubicados aquellos instrumentos, displays y sus controles asociados, que son indispensables para el vuelo seguro del avión, y las indicaciones luminosas como son las señales de alerta.

El campo de visión secundario (SFV por sus siglas en inglés) es el que se consigue mediante la rotación de la cabeza pero sin rotar los ojos. Dentro del SFV deben quedar ubicados aquellos controles y demás partes de la cabina que siguen en importancia para poder volar la aeronave, después de los mencionados en el PFV.

La Fig. 4 muestra claramente el PFV, el SFV y la combinación de ambos, que se obtiene mediante la rotación conjunta de cabeza y ojos, así como muestra también dentro de cada campo de visión sus partes óptima y máxima.

FIG. 4. CAMPOS DE VISIÓN PARA LOS TRIPULANTES

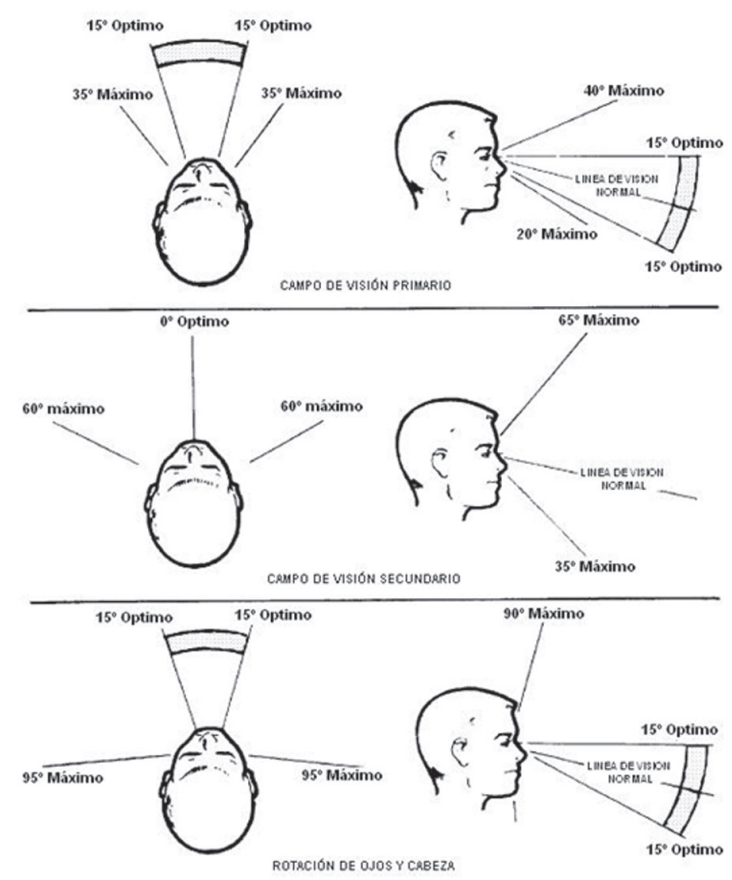

Fuente: Los autores

De acuerdo con lo anterior se trazaron los vectores que definen los campos el PFV y el SFV en los diagramas elaborados en AutoCAD ${ }^{\circledR}$ para los tres dummies y se hicieron los respectivos análisis de visibilidad interna, se busca verificar que los controles, instrumentos y displays se encuentren ubicados dentro del campo que corresponde.

\section{CONTROLES DE MANDO Y PANEL DE INSTRUMENTOS}

\section{A. Controles de mando}

En la actualidad existen varios tipos de controles de mando, los más usados en aviación general son de tipo columna central desde el panel, el mando lateral o sidestick y la columna central tipo joystick. La decisión de implementar uno u otro dispositivo de control de mando está basada en consideraciones de seguridad y ergonomía antes que por espacio interior de cabina.

La parte de seguridad involucra determinar si el control de mando se convierte en un elemento letal a la hora de un accidente donde se producen desaceleraciones súbitas de la aeronave. Para esto se trazan las trayectorias de impacto, la cuales muestran la interacción entre el desplazamiento del cuerpo debido a la desaceleración y el control de mando, tal como lo muestra la Fig. 5.

FIG. 5. TRAYECTORIA DE IMPACTO PARA TRIPULANTES

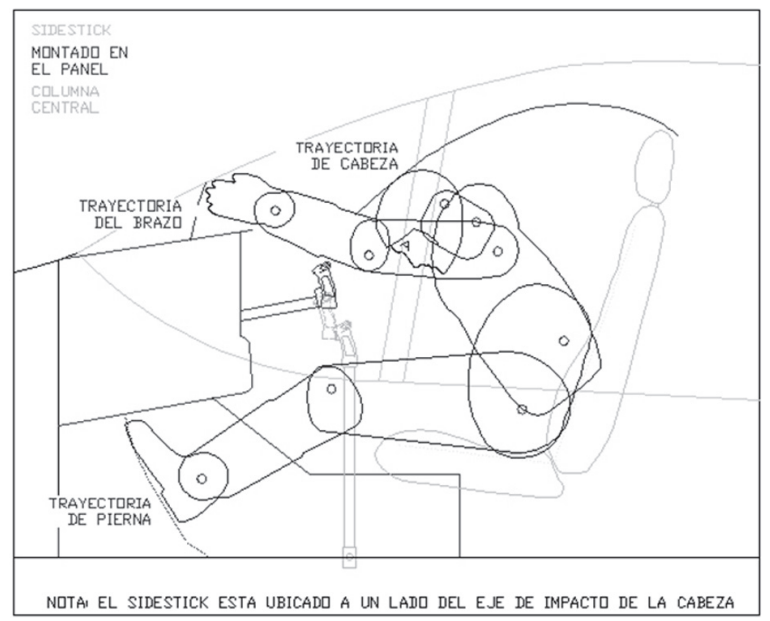

Fuente: Los autores

Respecto a la ergonomía, el análisis se realiza al ubicar las opciones de control de mando dentro de los diagramas de alcance mostrados en la Fig. 2 para piloto y copiloto, los cuales permiten determinar la facilidad con la que el tripulante puede manipular el control sin realizar esfuerzos adicionales. Esto se muestra en la Fig.6.

Aunque las tres opciones de controles de mando pueden ser alcanzada por el tripulante, ya que se encuentran dentro del diagrama de alcance, son las opciones de columna central las que presentan inconvenientes de seguridad y movilidad. La 
columna central desde el panel puede causar lesiones en la cabeza debido a su posición en el diagrama de impacto. De igual manera, el control central tipo joystick presenta problemas de espacio a la hora de ingreso a cabina y movilidad de las piernas del tripulante.

FIG. 6. UBICACIÓN DE CONTROLES DE MANDO

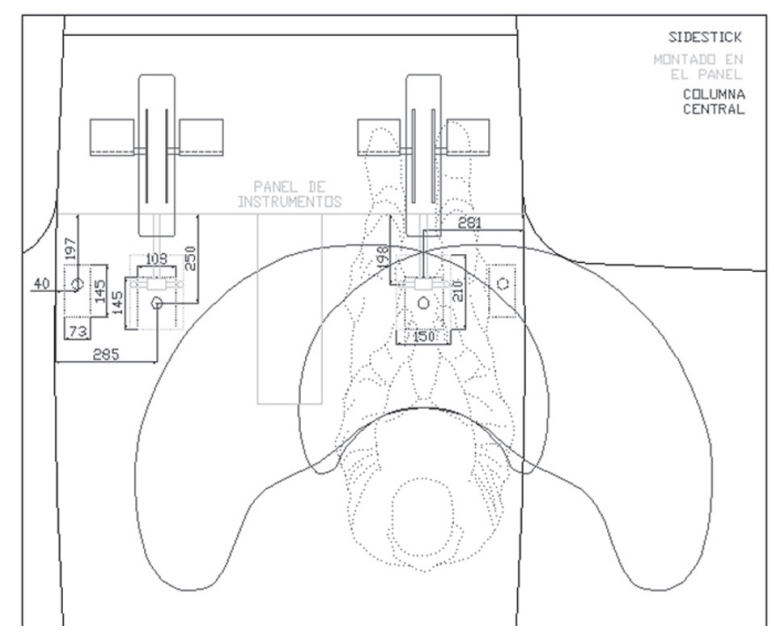

Fuente: Los autores

Una vez comparadas las tres opciones propuestas, se determina que el mando lateral o sidestick es el ideal para su uso como mando principal para el Phaeton. En términos de comodidad y confort del tripulante, este control permite tener acceso libre a la cabina y facilita la evacuación en una posible emergencia. Sin embargo, por razones de ergonomía es necesario un apoyabrazos que permitia movimientos de mayor precisión y sensibilidad a la hora de controlar la aeronave. Este apoyabrazos hace parte del cubrimiento interior de cabina y se extenderá según las líneas del fuselaje hasta un máximo de $500 \mathrm{~mm}$ de altura respecto al piso de cabina.

\section{B. Panel de instrumentos}

El panel de instrumentos juega un papel muy importante dentro de la cabina pues influye directamente en la carga de trabajo de los pilotos y determina la comodidad al momento de utilizar los instrumentos. Dentro de este análisis se consideran la distribución de la aviónica dentro del panel y la distancia entre éste y el tripulante.

La ubicación del panel está dada por la distancia para colocación de información visual a los instrumentos, en este caso a las pantallas de la aviónica digital. La FAA[5] recomienda una distancia máxima de $737 \mathrm{~mm}$, la cual permite una lectura adecuada para pantallas a partir de 10.4" y diales de un diámetro hasta de $76 \mathrm{~mm}$. Dado que los instrumentos analógicos usados tienen un diámetro de $80 \mathrm{~mm}$ y las pantallas son de 12.1", esta distancia permite una lectura correcta sin esfuerzo visual. En adición a esto, tal distancia queda dentro del diagrama de alcance del tripulante lo que le brinda más comodidad a la hora de interactuar con los instrumentos.

De acuerdo a los nuevos avances en aviónica digital, se determina implementar la Garmin ${ }^{\circledR}$ G1000 ${ }^{\circledR}$ de uso extendido en aeronaves en servicio de la misma categoría del Phaeton y cuya implementación ha sido positiva para los pilotos. Sin embargo, debido a consideraciones FAR 23 es necesario contar con un indicador de altitud, un velocímetro y un altímetro analógicos, los cuales serán eléctricos a 28VDC para ahorrar espacio y peso al eliminar el sistema de vacío convencional.

\section{Ergonomía de controles e instrumentos}

El análisis de ergonomía de controles e instrumentos depende de la localización y organización de estos de tal manera que la tripulación pueda ver, alcanzar y manipularlos de manera adecuada sin esfuerzo físico adicional, bajo cualquier condición de luz y de vuelo.

Con los mismos dummies modelados en Auto$C A D^{\circledR}$ para el dimensionamiento de cabina, se ubican estos en su posición de operación del sidestick, lo cual permite observar cuánta extensión del brazo debe hacer el piloto para mover en todo su recorrido el control de mando. Como resultado, el sidestick se ubica a una altura de $60,7 \mathrm{~cm}$ del piso y separado del panel frontal y de la pared lateral $25.5 \mathrm{~cm}$ y $4.0 \mathrm{~cm}$ respectivamente.

Para el caso de las palancas de potencia y mezcla; y los controles de flaps y compensadores, estos se ubicaron en el pedestal según los lineamientos de identificación FAR 23 y recomendaciones de la GAMA[6]. Luego de un proceso iterativo donde la comodidad de los tripulantes en las tres estaturas analizadas es la variable estudiada, los centros de las palancas de potencia quedan situados a una distancia de $89.5 \mathrm{~cm}$ desde la pared de fuego de la aeronave y a una altura de 
$17.5 \mathrm{~cm}$ desde el SRP en posición neutral. Para el caso de los controles de flaps y compensadores, estos quedan ubicados sobre superficies inclinadas del pedestal por encima de las palancas de potencia, tal como lo muestra la Fig. 7.

FIG. 7. DIMENSIONES DEL PEDESTAL EN CABINA

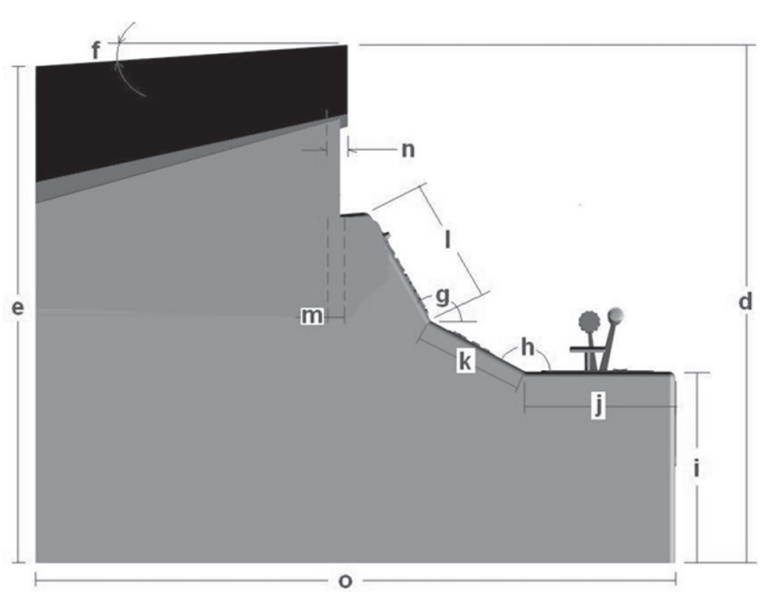

\begin{tabular}{|c|c|}
\hline Dimensión & Magnitud $(\mathrm{cm})$ \\
\hline $\mathrm{d}$ & 87 \\
\hline $\mathrm{e}$ & 83,5 \\
\hline $\mathrm{f}$ & $4^{\circ}$ \\
\hline $\mathrm{g}$ & $120^{\circ}$ \\
\hline $\mathrm{h}$ & $152^{\circ}$ \\
\hline $\mathrm{i}$ & 32 \\
\hline $\mathrm{j}$ & 24 \\
\hline $\mathrm{k}$ & 17,7 \\
\hline $\mathrm{l}$ & 20,2 \\
\hline $\mathrm{m}$ & 4,1 \\
\hline $\mathrm{n}$ & 5,7 \\
\hline $\mathrm{o}$ & 107,7 \\
\hline
\end{tabular}

Fuente: Los autores

Adicional a esto, el diseño del Phaeton ha seguido los lineamientos expuestos en las FAR 23 en miras de su certificación posterior. Es por esto que cada interruptor, perilla, instrumento y control que manipula la tripulación, sigue los métodos de identificación y dirección del movimiento definido por esta reglamentación, los cuales facilitan la disminución de la carga de trabajo de los pilotos. De igual manera, se siguen los lineamientos definidos por la GAMA[6] respecto a la retroalimentación de
Ios controles, es decir, como debe sentir la tripulación la interacción con estos dispositivos para dar una verdadera indicación de su funcionamiento.

\section{OTROS ANÁLISIS Y SELECCIÓN DE SUBSISTEMAS}

\section{A. Canopy y parabrisas}

La cubierta de Phaeton constará básicamente de dos grandes partes: el parabrisas frontal, fijamente anclado al fuselaje del avión; y la cubierta posterior que conforma el resto de concha para el compartimento de ocupantes, está formada por unos marcos en estructura metálica que proveerán rigidez a la cubierta y el resto estará conformado por material transparente que permita visibilidad hacia arriba, los laterales de pilotos y pasajeros y hacia atrás. Esta cubierta posterior está diseñada de manera que pueda deslizarse hacia adelante y atrás con el fin de abrir y cerrar la cabina, siendo la vía de acceso y de salida para el avión.

El anclaje del parabrisas se hará mediante un marco que sujeta al material transparente en su interior y que, a su vez, se fija al fuselaje mediante tornillos que permitan liberar fácilmente todo el conjunto al momento de hacerse un reemplazo. La sujeción del parabrisas al interior del marco de montaje se diseña de tal modo que sea posible el desplazamiento relativo del material transparente respecto al marco debido a las diferencias de expansión-contracción de ambos materiales frente a los cambios de temperatura.

Para satisfacer las consideraciones expuestas por la FAA[4] se opta por un diseño de marco que está conformado por una estructura metálica resistente en forma de canal, en cuyo interior se aloja el parabrisas pero con un recubrimiento de espuma o caucho que facilita el desplazamiento entre ambos materiales (transparente y metálico) y a la vez que los aísla del rozamiento entre sí, que alivia la presión de sujeción y permite los movimientos de flexión entre marco y parabrisas. Cortes del marco del parabrisas se presentan en la Fig. 8. Este diseño se basa en la instalación del parabrisas en una aeronave pequeña del tipo Piper PA-38 Tomahawk[2]. 
FIG. 8. CORTES DEL MARCO DEL PARABRISAS

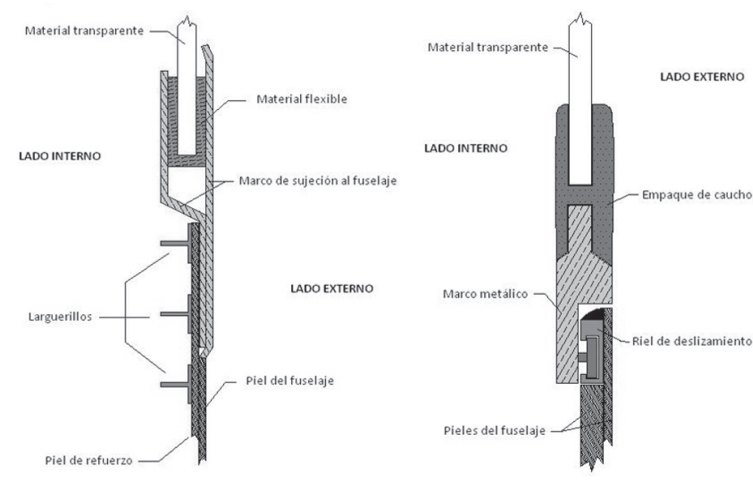

Fuente: Los autores

A diferencia del parabrisas, la parte posterior de la cubierta estará también anclada al fuselaje pero no de manera fija sino a través de un riel que permita deslizarla atrás y adelante para abrir y cerrar la cubierta.

Para el caso de volcamiento, el canopy proveerá protección de tal manera que la estructura de la cubierta no sufra una deformación tal que al momento del impacto contra el suelo, sea la cabeza del tripulante la que reciba el golpe directo. Por esto, los marcos de la cubierta incluirán refuerzos estructurales que permiten incrementar la rigidez del conjunto de cubierta. Estos refuerzos estructurales conforman una jaula antivuelco (roll cage) capaz de absorber energía de impacto y que asegure la integridad de la cubierta en caso de accidente.

El componente principal de la jaula, es el marco vertical del parabrisas justo donde se une con la cubierta posterior, dentro del cual se hace pasar un tubo de acero sujetado al fuselaje, que permite la disipación de fuerzas de impacto a la estructura principal de la aeronave. Debido a la forma compleja de la cubierta deslizante, los marcos de ésta serán sándwich de varias capas de lámina metálica y compuesta, se logra obtener la resistencia deseada y ayuda a dar el efecto de la jaula antivuelco a esta sección deslizante.

Para el caso en que la cubierta deslizante no pueda abrirse, las ventanas laterales serán del tipo expulsable. Este sistema permite darles la posibilidad a los tripulantes de empujar la ventana desde adentro y obtener una vía de evacuación. Esto se logra mediante la incorporación de un empaque de caucho alrededor de la ventana, el cual permite sujetarla a la estructura del canopy. El caucho, además de mantener sellada la ventana contra el fuselaje, deja un grado de libertad al momento de empujar la ventana, logrando que esta se desprenda del empaque y sea retirada. Este empaque, de sección transversal en forma de $\mathrm{H}$, sujeta al marco con uno de sus extremos y el otro sujeta el material transparente de la ventana(...). Ver Fig. 9.

En cuanto al material transparente usado en la cubierta de un avión se sugieren principalmente 5 materiales según FAA[4]: vidrio endurecido térmicamente, vidrio endurecido químicamente, acrílico fundido, acrílico estirado y policarbonato. Con el fin de determinar el mejor material en la cubierta del Phaeton se construyó una matriz de selección en la cual se comparan los 5 materiales mediante la calificación cuantitativa del comportamiento o características de cada uno frente a diferentes propiedades como, por ejemplo, la densidad y la resistencia al impacto. Una vez se hizo dicha calificación según el grado de importancia de cada propiedad analizada para el diseño de la cubierta, se concluyó que el material más apropiado para el Phaeton es el policarbonato por sus buenas características ópticas, baja sensibilidad al daño superficial, difícil propagación de fractura, alta resistencia al impacto, flexibilidad y buen comportamiento bajo fatiga entre otros; con los acrílicos como segunda opción.

\section{B. Asientos y sistemas de retención}

1) Asientos

El asiento hace parte fundamental del confort en cabina, pues brinda el soporte lumbar suficiente para efectuar los movimientos de las piernas para los pedales y un factor fundamental en evitar la fatiga de la tripulación.

Para realizar la selección de la silla en el Phaeton se tienen en cuenta las recomendaciones existentes acerca de las dimensiones y consideraciones de confort junto con datos recolectados en aeronaves del mismo tipo del Phaeton durante una visita de campo realizada al aeropuerto Enrique Olaya Herrera de Medellín.

Los asientos de cabina deben soportar al menos las cargas inerciales últimas para la aeronave en caso de desaceleración horizontal o vertical. Esto implica que la estructura interna, la estructura de anclaje al piso del avión y la cojinería 
de la silla soporten y amortigüen dichas cargas, para proteger la vida del ocupante.

La espuma que se usa en los asientos ha de tener la capacidad de absorber el impacto y ayudar a la disipación de energía en lugar de capturarla y amplificarla.

Como otras medidas de seguridad, los cojines del asiento deben poseer capacidades de flotabilidad en caso de amarizajes, además de ser no inflamable y no tóxico en caso de incendios en cabina.

De acuerdo con las dimensiones que se han definido para los asientos del Phaeton en el diseño preliminar de su cabina, basados tanto en las recomendaciones de ergonomía como en los datos tomados durante la salida de campo en el Olaya Herrera, se procede a hacer una selección del asiento por comparación y análisis de propiedades de las opciones ofrecidas por algunos proveedores de estos productos en el mercado de aviación.

Una vez realizada la búsqueda de información sobre asientos para aeronaves de aviación general en Internet, se encontró como mejor opción la ofrecida por Oregon Aero ${ }^{\mathbb{B}}$ : la High-G ${ }^{\mathbb{R}}$ Seat[7].

\section{2) Sistemas de Restención para la Tripulación}

Para una orientación del ocupante sentado mirando al frente en una silla no eyectable, el sistema de retención más comúnmente usado y que es aceptado como sistema estándar para la tripulación por el ejército de los Estados Unidos es el sistema de arnés con doble correa de hombros, cinturón de seguridad y correa central de amarre a la silla o tiedown strap la cual se resiste, a la fuerza tirante hacia arriba de las correas de hombro previniendo así que el cinturón se desplace hasta la parte abdominal, lo cual causaría incomodidad en el usuario y, en caso de accidente, provocaría fuertes lesiones en dicha área puesto que allí no hay algún componente óseo que resista la presión ejercida por la correa durante la desaceleración repentina. Este es el sistema básico, sin embargo se han hecho variaciones sobre el mismo para mejorar la resistencia al movimiento lateral adicionando las Ilamadas correas reflejadas, y reemplazando las correas de hombro por un arnés de hombro ensamblado tipo collar el cual consiste en un material acolchado en forma de collar que se ajusta alrededor del cuello del piloto. Sobre este material se enrutan las correas de hombro, quedando así el acolchado entre el cuerpo y la correa.

Cada correa reflejada sale del riel de inercia ubicado atrás del piloto y se conecta al collar pasando a través de un rodillo para luego dirigirse nuevamente hacia atrás siendo fijada en el lado opuesto de la correa de hombro por la cual fue pasada, en el respaldo de la silla. En otras palabras, la correa reflejada que sale del riel y se conecta a la correa del hombro derecho tiene su otro extremo fijo en algún punto duro en el respaldo del asiento, pero al lado izquierdo.

FIG. 9. SISTEMA DE RETENCIÓN MODIFICADO

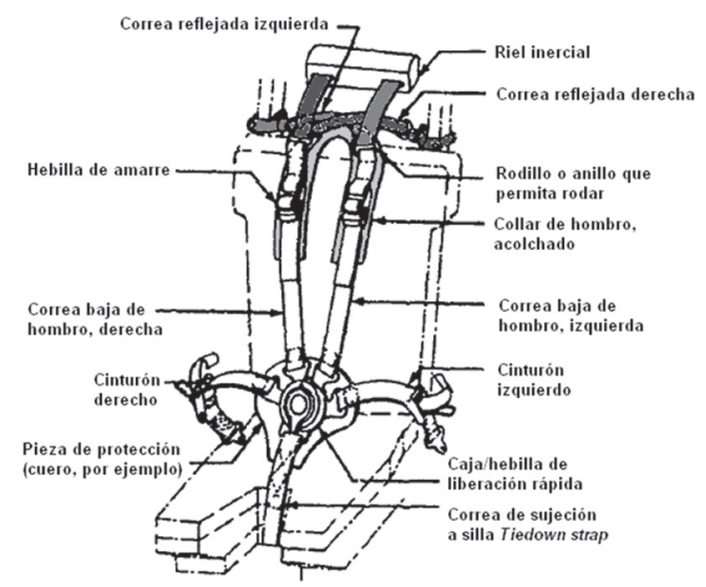

Fuente: Los autores

Este sistema, en cualquiera de sus dos formas, básico o con las adiciones mencionadas, tiene una única hebilla de sujeción-liberación que va permanentemente fija a la tiedown strap y al cual se conectan las demás correas en sus respectivos puntos, como lo muestran la Fig. 9 correspondiente al sistema modificado con las correas reflejadas y el arnés de tipo collar.

Estos dos sistemas de retención: el básico o el modificado son los seleccionados para usar en la cabina del Phaeton.

\section{Consideraciones de confort y seguridad en cabina}

El confort en una cabina de una aeronave depende de la comodidad física y de la carga de trabajo que tenga la tripulación en las fases críticas del vuelo. Carga de trabajo es un término usado para describir la relación entre la capacidad de un individuo para realizar una tarea (física y/o mental), 
y el nivel de demanda situacional y del sistema asociado con el llevar a cabo esa tarea. Este factor junto a la fatiga del piloto son los principales responsables del detrimento del desempeño de una tripulación, que aumentando el potencial error humano.

La razón del uso de aviónica digital dentro de la aeronave es porque estos nuevos sistemas cambian la apariencia, operación y usabilidad de la interface piloto-maquina, e incrementan la seguridad y la alerta situacional de la tripulación. Adicionalmente, el tiempo de respuesta para la lectura de un display digital es inferior al de un display analógico, que convierte el digital en el óptimo para descargar la carga de trabajo del piloto, pues no tendrá que añadir el cálculo de las posiciones de los indicadores respecto a las marcas del instrumento a su lista de procesos mentales en vuelo.

Para el caso del Phaeton, la plataforma donde el diseño está fundamentado es la estandarización. Esta juega un papel importante en la determinación de la carga de trabajo de un piloto pues permite una rápida familiarización, no sólo con la configuración de diseño sino con las demás cabinas que sostengan el mismo estándar. Sin embargo, la forma de encontrar el punto medio donde el piloto está comprometido en el proceso para mantenerse alerta e involucrado en la operación del sistema sin tener exceso de carga, es la experimentación, por lo que sólo una vez terminada la aeronave será posible determinar el comportamiento de la tripulación en ambientes de operación típicos.

\section{1) Sistemas de control de ambiente}

Phaeton está provisto de un control climático con acceso remoto en cabina, ubicado en el lado derecho del panel de instrumentos, el cual permite mantener la calefacción y enfriamiento para la tripulación. Para tal propósito se considerarán dos tipos de sistemas de control de ambiente: un sistema de ventilación y calefacción basado en aire de impacto y un sistema de acondicionamiento de aire por ciclo de vapor.

En el sistema estándar de la aeronave, que emplea aire de impacto para renovar el ambiente en la cabina el aire es forzado por un ventilador hacia un distribuidor principal que lo reparte hasta las salidas de aire en cabina. El sistema opcional de acondicionamiento por ciclo de vapor igual emplea aire de impacto, sin embargo, existe un sistema de refrigeración mediante tuberías de R134a amigable al ambiente, el cual enfría el aire y lo mantiene a las condiciones de temperatura deseada en cabina.

Todo el control sobre la temperatura, el volumen de aire y selección del flujo es llevado a cabo con la manipulación de los selectores de flujo de aire, temperatura y dirección del aire en el control de cabina, localizado junto a las pantallas de aviónica en el lado derecho del panel de instrumentos. Este control se realiza mediante indicadores de fácil identificación.

\section{2) Equipos de emergencia a bordo}

Dos nuevas tecnologías en el mundo de la aviación serán consideradas en este diseño para incrementar la seguridad tanto de tripulación como pasajeros. Estas tecnologías son introducidas en la aviación general por grandes fabricantes como Cessna, Beechcra$\mathrm{ft}$ y Cirrus, entre otros; y son apropiadas por las demás aeronaves actualmente en servicio. Con una significativa reducción de fatalidades en los accidentes ocurridos en los últimos años, el uso de los airbags y el paracaídas de emergencia (spin parachute) está considerado como dispositivos de seguridad estándar por las autoridades aeronáuticas mundiales.

Airbags son superficies inflables que se despliegan desde el sistema de restricción de tripulantes y pasajeros, las cuales crean una barrera de protección delante de la persona en caso de desaceleraciones abruptas que puedan llevar a golpearse contra elementos de la cabina.

\section{3) Spin parachute}

Previene a la aeronave de pérdidas de control en maniobras fuera de lo común o en condiciones de pérdida por actitudes extrañas de la aeronave causadas por error humano o condiciones meteorológicas adversas. Este dispositivo se encuentra ubicado en un compartimiento en el fuselaje justo detrás de la cabina de vuelo, el cual es desplegado mediante un cohete propulsor que lo lleva a la altura óptima de despliegue. La activación va a discreción del piloto mediante una palanca en cabina, la cual energiza el sistema completo que produce el despliegue del paracaídas. 
Este dispositivo es considerado dentro del diseño del Phaeton con el fin de proveerle más seguridad a la aeronave.

\section{AGRADECIMIENTOS}

Los autores reconocen las contribuciones de los ingenieros Jorge Iván García Sepúlveda y Juan Pablo Alvarado Perilla como director y asesor respectivamente del trabajo del cual se derivó el presente artículo, así como también agradece las contribuciones de los ingenieros Daniel E. Restrepo y Herman Mauricio Pérez.

\section{REFERENCIAS}

[1] ESTRADA M. Jairo y otros. Parámetros Antropométricos de la Población Laboral Colombiana 1995 (Acopla95). En: Revista Facultad Nacional de Salud Pública. Medellín. Vol. 15, No. 2 (Ene. - Jun. 1998); p. 112-139.

[2] ROSKAM, Jan. Airplane Design Part III: Layout Design of Cockpit, Fuselage, Wing and Empennage: Cutaways and Inboard Profiles. Lawrence, KA: DARcorporation, 2002. 454 p.

[3] FEDERAL Aviation Administration. Advisory Circular 25.773-1: Pilot Compartment View Design Considerations. S.I.: FAA, 1993. 6 p.

[4] FEDERAL Aviation Administration. Advisory Circular 25.775-1: Windows and Windshields. S.I.: FAA, 2003. $10 \mathrm{p}$.

[5] FEDERAL Aviation Administration Human Factors Division. Human Factors Criteria for Displays: A Human Factors Design Standard Update of Chapter 5. Washington, DC: FAA, 2007. p. 5-7

[6] GENERAL AVIATION MANUFACTURERS ASSOCIATION. Recommended Practices and Guidelines for Part 23 Cockpit/Flight Deck Design. Washington, DC: GAMA, 2000. $103 p$.

[7] OREGON Aero ${ }^{\circledR}$. Oregon Aero ${ }^{\circledR}$ High-G® Seat. <http://www.oregonaero.com/p48_2001.htm> 